\title{
Análise e melhoria do processo de reengenharia de software: um estudo de caso
}

\author{
Guilherme Mendonça de Moraes', Prof. Dr. Edgard Costa Oliveira² \\ ${ }^{1}$ Programa de Pós-Graduação em Computação Aplicada (PPCA) - Universidade de \\ Brasília (UnB), CEP: 7090-970 - ICC Centro, Módulo 14, Subsolo CSS 361, Campus \\ Universitário Darcy Ribeiro - Brasília, DF - Brasil \\ ${ }^{2}$ Faculdade de Tecnologia - Engenharia de Produção (FT/ERP) - Universidade de \\ Brasília (UnB), DF - Brasil. \\ guilherme.moraesdaluno.unb.br, ecosta@unb.br
}

\begin{abstract}
Although many authors seek to relate risk management to failure or success in software reengineering projects, the union of the two themes remains a major challenge for organizations that contract or provide such products and services. This article aims to analyze and improve the process of software reengineering through risk management. The methodology adopted was the case study of a project to change the technology of software in a large financial institution, which resulted in the improvement of the software reengineering process in the company.
\end{abstract}

Resumo. Apesar de muitos autores procurarem relacionar gestão de riscos com fracasso ou sucesso em projetos de reengenharia de software, a união das duas temáticas ainda é um grande desafio para as organizações que contratam ou fornecem produtos e serviços desse tipo. Esse artigo tem por objetivo a análise e melhoria do processo de reengenharia de software por meio da gestão de riscos. A metodologia adotada foi o estudo de caso de um projeto de mudança da tecnologia do software em uma instituição financeira de grande porte, o que resultou na melhoria do processo de reengenharia de software dessa instituição.

\section{Introdução}

Com o avanço tecnológico e o crescimento das necessidades de mercado, é comum que as empresas optem por realizar a mudança de sua estrutura tecnológica atual visando a compatibilidade com outras linguagens, banco de dados e plataformas, como também o aumento do desempenho, usabilidade, acessibilidade, segurança e qualidade [Pinto e Braga, 2004; UTFPR, 2010; Machado, 2011].

A mudança da tecnologia visa não somente adaptar o processo de software ao de negócio [Weerakkody e Currie, 2003], mas também adequá-lo às novas tecnologias, corrigindo erros conhecidos em ambiente produtivo, como itens de qualidade expostos pela ISO 25010 (2011): funcionalidade, confiabilidade, usabilidade, eficiência, manutenibilidade e portabilidade, fazendo com que o aplicativo cumpra requisitos específicos em tempo real [Vogel-Heuser et al., 2014]. 
De acordo com Pressman (2005) essa atividade de reengenharia de sistemas de computacionais consome até $70 \%$ dos esforços durante todo o ciclo de vida do sistema.

Além da reengenharia de software ser uma tarefa onerosa, existe um alto risco nesse processo de mudança da tecnologia [Pressman , 1995; Reis et al., 2003; Vogel-Heuser, 2010], pois o sistema legado precisa continuar funcionando sem que essas modificações causem um efeito negativo no mesmo em ambiente produtivo.

Juntamente com essas alterações existem os riscos que as mesmas representam para o negócio da instituição [Weerakkody e Currie, 2003; Sneed, 2005; Chen et al., 2009], pois a necessidade é que a toda a estrutura dos aplicativos seja alterada sem que o programa em produção fique comprometido.

Conforme a ISO 31000 (2009) risco é o efeito da incerteza nos objetivos. Em outras palavras, é algo que pode causar um desvio nos propósitos comuns. Nesse contexto, o objetivo está relacionado às expectativas de qualidade, funcionamento correto e conformidade com requisitos legais e institucionais do software migrado, tendo como base que suas vulnerabilidades estão ligadas diretamente à ausência de determinados controles [Elahi et al., 2010].

Dessa forma, o presente trabalho tem por objetivo a análise e melhoria do processo de reengenharia de software desenhando o processo atual $(A S-I S)$, analisando as vulnerabilidades do processo, propondo um desenho do mesmo (TO-BE) com as melhorias utilizando ferramentas e técnicas para gestão de riscos.

O estudo de caso em questão será aplicado a um projeto de mudança da tecnologia de aplicativos computacionais em uma instituição financeira de grande porte, que por sigilo terá sua identificação nesse artigo como Instituição Financeira Creditar.

\section{Desenho do processo atual $(A S-I S)$ de reengenharia de software}

Devido à falta de gestão de riscos nos processo de migração da tecnologia do software, a Instituição Financeira Creditar reportou um incidente em ambiente produtivo após migração de um aplicativo computacional e o mesmo tendo passado por todas as atividades de desenvolvimento, teste e homologação, que creditou várias vezes o salário de clientes, que por sua vez causou um grande preiuízo financeiro. dano à imagem da instituição:

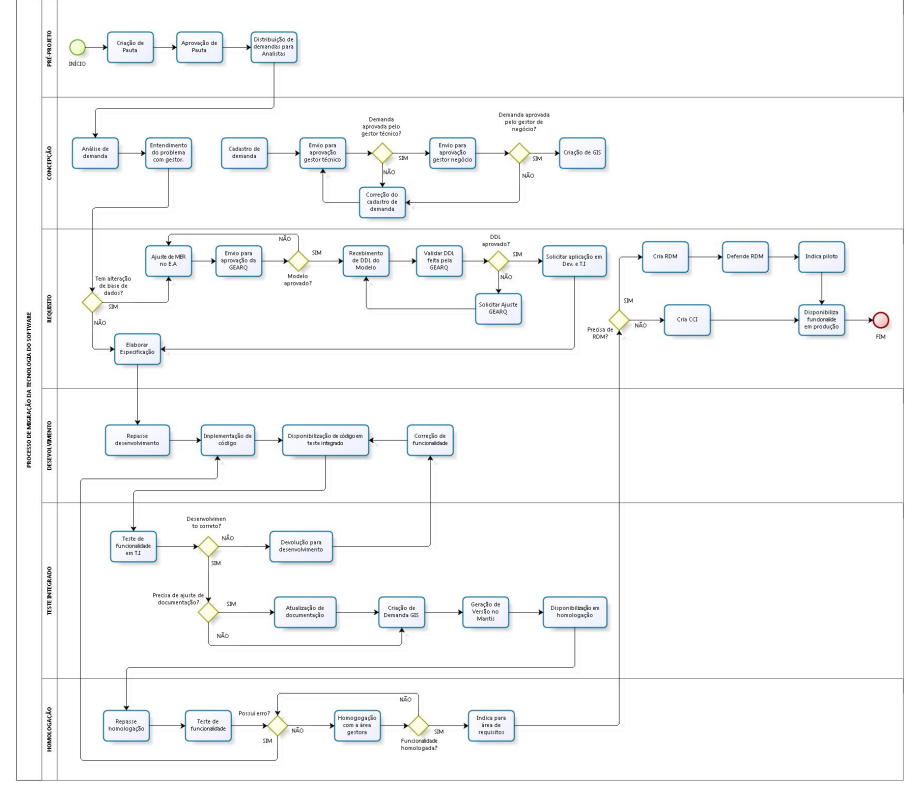


Utilizando a técnica de mapeamento de processos BPMN - Business Process Model and Notation [Chinosi e Trombetta, 2012] e o programa Bizagi, realizado o desenho AS-IS do processo de migração da tecnologia de software onde o mesmo foi dividido em 6 grandes áreas sendo: pré-projeto, concepção, requisitos, teste integrado e homologação conforme imagem anterior.

\section{Identificação dos riscos no processo atual de reengenharia de software}

Como a proposta da ISO 31000 (2009) é a identificação, análise, avaliação e tratamento dos riscos, é necessário em primeiro ponto realizar a identificação dos mesmos relacionado à todas as atividades da organização. Para tal tarefa, foi utilizada a técnica Brainstorming que é uma ferramenta indicada pela ISO 31010 (2009) e uma forma obter uma lista completa dessas ameaças. Após tais tarefas, foi construído um desenho com uma estrutura da análise SWOT - Strengths, Weaknesses, Oportunities, Thereats, porém, considerando apenas as oportunidades e ameaçadas identificadas no Projeto Renovação CCO da instituição estudada:

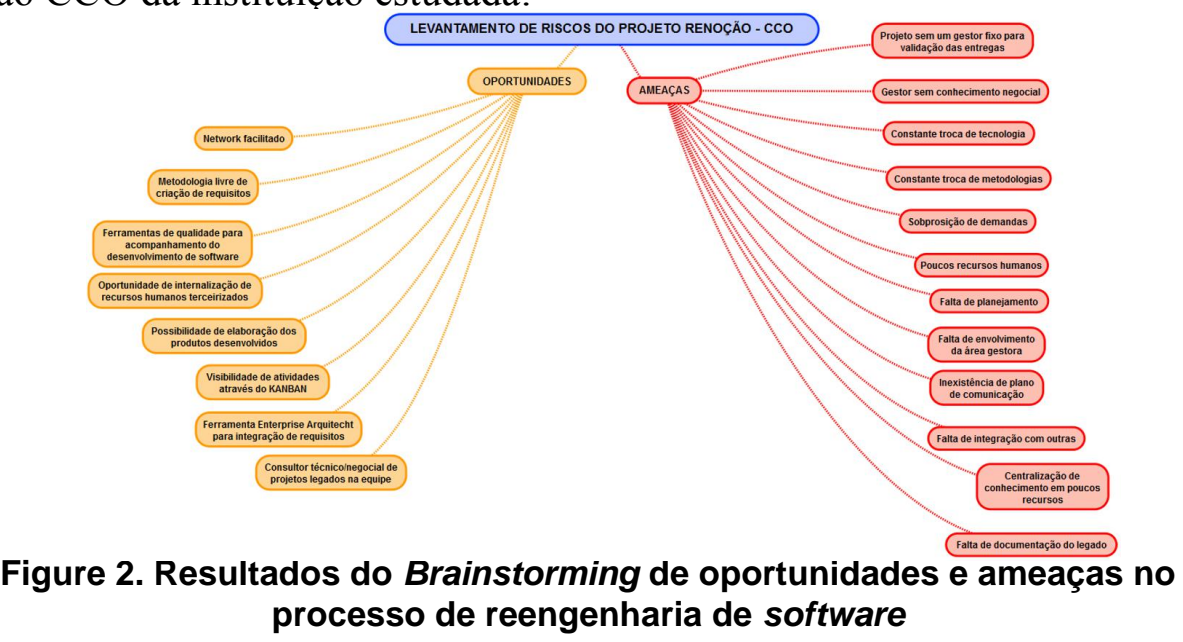

Para identificar o que foi considerado como ameaça, foram utilizados ferramentas, técnicas e melhores práticas para reengenharia de software e serviços de TI como: Rational Unifield Process [IBM, 2001], SCRUM [Schwaber e Sutherland, 2011], Xtreme Promming [Beck e Gamma, 2000], ITIL [OGC, 2011], ISO 25010 (2011) e o PMBOK (2018) e com isso criada uma base de conhecimento após essa revisão bibliográfica para identificar os controles aplicáveis às vulnerabilidades no processo de reengenharia de aplicativos do estudo de caso, como a seguir:

\section{Quadro 1. Modelo CGU (2018) de base de conhecimento com a documentação} dos controles

\begin{tabular}{|l|l|l|}
\hline \multicolumn{1}{|c|}{$\begin{array}{c}\text { Aplicação: Gestão de } \\
\text { Projetos }\end{array}$} & $\begin{array}{c}\text { Ativo: } \\
\text { Tecnologia }\end{array}$ Tipo: Norma $\quad$ Knowledge base: ISO 25010 (2011) \\
\hline Descrição do controle & $\begin{array}{l}\text { Deve-se criar um documento de Interoperabilidade. } \\
\text { O quê? }\end{array}$ & $\begin{array}{l}\text { Artefato para documentar a capacidade do produto de software de } \\
\text { interagir com um ou mais sistemas especificados. }\end{array}$ \\
\hline Por quê? & $\begin{array}{l}\text { Mitigação dos riscos de erro de integração do novo sistema migrado com } \\
\text { outros softwares necessários. }\end{array}$ \\
\hline
\end{tabular}


Após o Brainstorming e com ajuda de gestores do negócio, algumas partes interessadas e um gestor de riscos, chegou-se ao conhecimento da existência de cerca de 150 vulnerabilidades em todo o processo de reengenharia de software, das quais foram selecionadas 25 para análise, avaliação e tratamento, sendo elas de maior relevância para a instituição de acordo com os especialistas envolvidos no nessas atividades.

Foram utilizadas como referência a norma ISO 25010 (2011), PMBOK (2018), o RUP [IBM, 2001], ITIL V3 [OGC, 2011], Xtreme Promming [Beck e Gamma, 2000] e o SCRUM [Schwaber e Sutherland, 2011] para identificação dos controle aplicáveis às vulnerabilidades no processo de mudança da tecnologia do software. Com isso, foi realizada a documentação dos riscos como tais como esses:

\section{Quadro 2. Identificação de riscos com a especificação do impacto e aplicação dos controles (Adaptado pelo próprio autor do modelo CGU (2018))}

\begin{tabular}{|c|c|c|c|}
\hline Etapa & $\begin{array}{l}\text { Vulnerabilidades - } \\
\text { Fatores de Risco }\end{array}$ & Impacto & Controles Aplicáveis \\
\hline \multirow{6}{*}{$\begin{array}{l}\text { Pré- } \\
\text { projeto }\end{array}$} & \multirow{6}{*}{$\begin{array}{l}\text { Divergência de } \\
\text { planejamento e } \\
\text { execução de pauta e } \\
\text { especificação das } \\
\text { necessidades e } \\
\text { expectativas de cada } \\
\text { projeto }\end{array}$} & Mudanças não mapeadas & \multirow{6}{*}{$\begin{array}{l}\text { É necessário identificar todas as } \\
\text { necessidades, premissas, restrições, } \\
\text { requisitos iniciais e principais responsáveis } \\
\text { pelo projeto de forma a mitigar o risco de } \\
\text { mudança de escopo, aumento do escopo, } \\
\text { aumento de custo e prazo. }\end{array}$} \\
\hline & & $\begin{array}{l}\text { Falta de compatibilidade com } \\
\text { outras funcionalidades }\end{array}$ & \\
\hline & & $\begin{array}{l}\text { Erros desconhecidos em ambiente } \\
\text { produtivo }\end{array}$ & \\
\hline & & $\begin{array}{l}\text { Descumprimento de requisitos } \\
\text { negociais }\end{array}$ & \\
\hline & & Aumento do custo do projeto & \\
\hline & & Atraso no projeto & \\
\hline \multirow{6}{*}{ Concepção } & \multirow{6}{*}{$\begin{array}{l}\text { Falta de } \\
\text { entendimento do } \\
\text { sistema legado e } \\
\text { todas as } \\
\text { funcionalidades } \\
\text { internas e externas } \\
\text { impactadas por } \\
\text { determinada } \\
\text { mudança como } \\
\text { também os erros } \\
\text { existentes. }\end{array}$} & $\begin{array}{l}\text { Erros desconhecidos em ambiente } \\
\text { produtivo }\end{array}$ & \multirow{2}{*}{$\begin{array}{l}\text { Deve-se criar um documento de } \\
\text { Interoperabilidade para documentar a } \\
\text { capacidade do produto de software de } \\
\text { interagir com um ou mais sistemas } \\
\text { especificados. }\end{array}$} \\
\hline & & $\begin{array}{l}\text { Atualização desnecessária de } \\
\text { software ou nova funcionalidade }\end{array}$ & \\
\hline & & $\begin{array}{l}\text { Erros desconhecidos em ambiente } \\
\text { produtivo }\end{array}$ & \multirow{2}{*}{$\begin{array}{l}\text { Deve-se criar um documento de } \\
\text { Capacidade do produto de software de } \\
\text { prover um conjunto apropriado de funções } \\
\text { para tarefas e objetivos do usuário } \\
\text { especificados. }\end{array}$} \\
\hline & & Insatisfação do usuário final & \\
\hline & & Aumento do custo do projeto & \multirow{2}{*}{$\begin{array}{l}\text { Deve-se realizar a proteção frente a erros } \\
\text { de usuários: como produto consegue } \\
\text { prevenir erros dos usuários. }\end{array}$} \\
\hline & & Aumento do prazo do projeto & \\
\hline
\end{tabular}

\section{Análise e avaliação dos riscos no processo de reengenharia de software}

Com a utilização das ferramentas e técnicas: Análise SWOT e Análise de Restrição e Premissas [PMBOK, 2018], o gestor negocial da instituição estudada, juntamente com o gestor de riscos conseguiram identificar qual o grau de riscos de vulnerabilidade pontuando a probabilidade de determinado risco se concretizar como também o impacto que o mesmo causaria ao processo e mudança da tecnologia dos sistemas. Essas informações foram documentadas em uma matriz de probabilidade e impacto [ISO $31010,2011]$ na qual foi utilizada uma pontuação de probabilidade de 0,1 a 0,9 , sendo 0,1 para pouco provável e 0,9 para muito provável que determinado risco se torne um incidente, e no caso do impacto de 1 a 5 , sendo 1 para impacto muito baixo e 5 para 
impacto muito alto, caso o incidente aconteça. Para fins de cálculos, os valores foram multiplicados entre si gerando uma classificação de score com o grau de cada risco:

Tabela 1. Avaliação dos riscos (próprio autor adaptado da ISO 31010, 2009)

\begin{tabular}{|l|r|r|c|}
\hline \multicolumn{2}{|c|}{ AVALIAÇÃO DOS RISCOS - MATRIZ DE PROBABILIDADE E IMPACTO } \\
\hline \multicolumn{1}{|c|}{ Riscos } & Probabilidade & Impacto & Score \\
\hline Mudanças não mapeadas & 0,7 & 5 & 3,5 \\
\hline Falta de compatibilidade com outras funcionalidades & 0,7 & 5 & 3,5 \\
\hline Erros desconhecidos em ambiente produtivo & 0,3 & 4 & 1,2 \\
\hline Descumprimento de requisitos negociais & 0,1 & 2 & 0,2 \\
\hline Aumento do custo do projeto & 0,9 & 2 & 1,8 \\
\hline Atraso no projeto & 0,9 & 3 & 2,7 \\
\hline Erros desconhecidos em ambiente produtivo & 0,3 & 5 & 1,5 \\
\hline $\begin{array}{l}\text { Desenvolvimento desnecessário de atualização de software } \\
\text { ou nova funcionalidade }\end{array}$ & 0,2 & 1 & 0,2 \\
\hline Insatisfação do usuário final & 0,4 & 5 & 2 \\
\hline Aumento do custo do projeto & 0,3 & 3 & 0,9 \\
\hline
\end{tabular}

O score foi avaliado tendo como base a matriz de probabilidade e impacto [PMBOK, 2018], considerando apenas as ameaçadas:

Tabela 2. Matriz de Probabilidade e Impacto (Adaptado de PMBOK (2018))

\begin{tabular}{|c|c|c|c|c|c|}
\hline \multicolumn{7}{|c|}{ Matriz de Probabilidade X Impacto } \\
\hline Probabilidade & \multicolumn{5}{|c|}{ Ameaças } \\
\hline 0,9 & 0,9 & 1,8 & 2,7 & 3,6 & 4,5 \\
\hline 0,7 & 0,7 & 1,4 & 2,1 & 2,8 & 3,5 \\
\hline 0,5 & 0,5 & 1 & 1,5 & 2 & 2,5 \\
\hline 0,3 & 0,3 & 0,6 & 0,9 & 1,2 & 1,5 \\
\hline 0,1 & 0,1 & 0,2 & 0,3 & 0,4 & 0,5 \\
\hline Gravidade & 1 & 2 & 3 & 4 & 5 \\
\hline
\end{tabular}

\section{Proposta de melhoria no processo de reengenharia de software (TO-BE)}

Após identificação, análise e avaliação de risco e aplicação dos controles às vulnerabilidades identificadas no processo mudança da tecnologia do software, como resposta a essas ameaças encontradas no anterior $(A S-I S)$, foi elaborado um desenho evoluído do mesmo (TO-BE) como forma de mitigar os riscos identificados anteriormente, como por exemplo:

1. Na forma de elicitação de requisitos, foi identificado a necessidade de entender o sistema legado através de documentações existentes e principalmente a opinião de especialistas técnicos por Brainstorming e à partir daí, a elaboração de uma primeira documentação contendo as possíveis regras, telas (se aplicável) e entidades de banco de dados (se aplicável); 
2. Como documentação principal de mapeamento de necessidade e desenho de funcionalidades e suas respectivas regras, foi utilizado o conceito de elaboração de interface adaptando a ideia do RUP [Kruchten, 2004; Sommerville, 2011] de forma que todos os stakeholders consigam opinar sobre a viabilidade, necessidades e necessidades do negócio inerente as funcionalidade migrada;

3. Processo de validação de regras, mensagens, telas (quando aplicável) e entidades de banco de dados (quando aplicável) - é cíclica iniciando no gestor técnico, equipe de desenvolvimento, representando do usuário final do software migrado e por último o gestor do negócio, fazendo cada vez mais a documentação e detalhamento do serviço/sistema [ITIL, 2007].

A seguir, o desenho do processo melhorado $(T O-B E)$ :

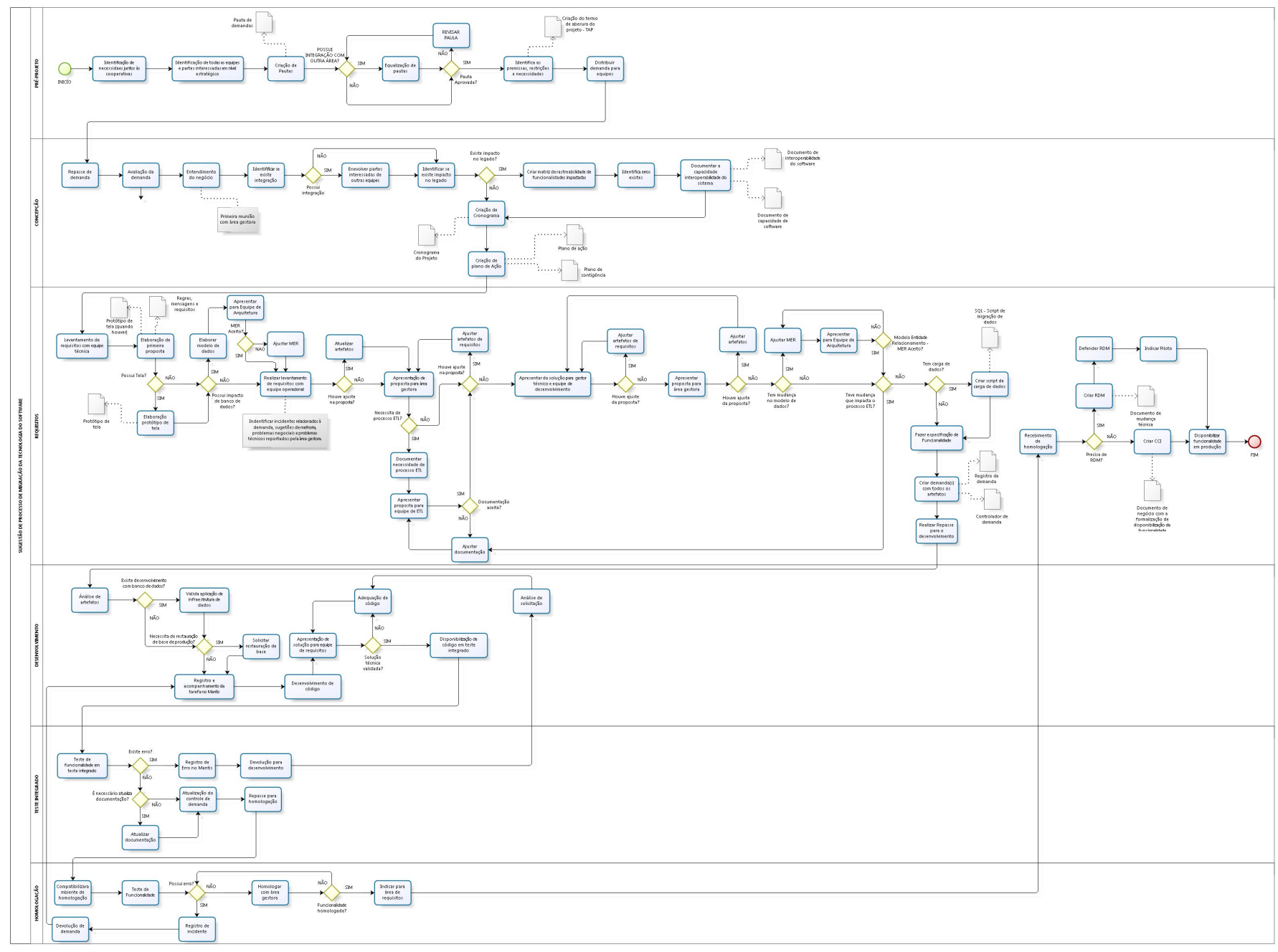

Figure 3. Desenho TO-BE do processo reengenharia de software da Instituição Financeira Creditar

Através do desenho do projeto melhorado, a área gestora pôde ter uma visão de quais atividades podem ser aplicadas para mitigar os riscos identificados no mapeamento anterior, tendo em vista que a versão aprimorada apresenta ações e documentos para reengenharia de software baseadas em controles de mercados como boas práticas e normas. 


\section{Conclusão}

O estudo em questão buscou propor a aplicação da gestão de riscos como forma de análise e melhoria do processo de reengenharia de software, mapeamento ferramentas e técnicas para identificar, analisar, avaliar e tratar os riscos que permeiam esse processo, como também sendo as respostas à esses riscos o processo já melhorado utilizando boas práticas, normas e frameworks.

Foram utilizas técnicas como brainstorming, matriz de probabilidade e impacto, mapeamento de processos, apoio de especialistas assim como na revisão da literatura para identificar os pontos de atenção e riscos no processo de reengenharia de software e como forma de resposta a esses riscos na elaboração do processo melhorado TO-BE.

Foi utilizado como análise um projeto de mudança de tecnologia do software da instituição financeira em questão que possui todas as características necessárias para geração de insumo da pesquisa em questão, que além das ferramentas e técnicas, contou com o apoio de um especialista de riscos e um gestor organizacional para validação do processo melhorado. O resultado final mostrou-se bem eficiente após validação do gestor de negócio e do especialista de risco, pois conseguiu revelar os riscos que o processo de reengenharia de software como também mostrou as vulnerabilidades que o processo possuía, ou seja, os pontos de atenção que o, por meio de um processo melhorado, permitiu a redução dos riscos de mudança, do não cumprimento de requisitos e do não atendimento de prazo e custos e até mesmo da extinção do projeto.

Após criação e aplicação do processo melhorado, observou-se a redução dos riscos de erros em ambiente produtivo dos softwares migrados, menor tempo de desenvolvimento, menos mudanças nos requisitos e funcionalidades e em consequência disso, diminuição do tempo e custo de desenvolvimento de todo o processo. Como recomendação para trabalhos futuros, propomos a aplicação de outras ferramentas e técnicas para gestão do risco como também uma forma automatizada para análise e avaliação do riscos identificados.

\section{Referências}

ABNT ISO GUIA 31.000:2009, Gestão de Riscos - Princípios e diretrizes, 1. ${ }^{a}$ edição, Rio de Janeiro, ABNT, 2009.

ABNT ISO GUIA 31.010:2012, Gestão de Riscos - Técnicas para o processo de avaliação de riscos, $1^{\text {a }}$ edição, Rio de Janeiro, ABNT, 2012.

Beck, K., \& Gamma, E. (2000). Extreme programming explained: embrace change. addison-wesleyprofessional.

Cagnin, M. I. (2005). Parfait: uma contribuição para a reengenharia de software baseada em linguagens de padrões e frameworks (Unpublished doctoral dissertation). Universidade de São Paulo.

Chaves, L. L. (2004). Sistemas legados e a aplicação de processos de reengenharia de software. FEA - USP , 21-23.

Chen, C. C., Law, C. C., \& Yang, S. C. (2009). Managing ERP implementation failure: a project management perspective. IEEE transactions on engineering management, $56(1), 157-170$. 
Chinosi, M., \& Trombetta, A. (2012). BPMN: An introduction to the standard. Computer Standards \& Interfaces, 34(1), 124-134.

De Bakker, K., Boonstra, A., \& Wortmann, H. (2010). Does risk management contribute to it project success? a meta-analysis of empirical evidence. International Journal of Project Management, 28 (5), 493-503.

Elahi, G., Yu, E., \& Zannone, N. (2010). A vulnerability-centric requirements engineering framework: analyzing security attacks, countermeasures, and requirements based on vulnerabilities. Requirements engineering, 15(1), 41-62.

Ibbs, C. W., \& Kwak, Y. H. (2000). Assessing project management maturity. Project management journal, 31 (1), 32-43.

ISO ISO/IEC 25.010 (2011), Software Product Quality. International Organization for Standardization.

ISO/IEC 13.335-1 (2004) Information technology - Security techniques - Management of information and communications technology security.

ITIL V3 - Information Technology Infrastructure Library (2011). OGC (Office for Government Commerce.

Kruchten, P. (2004). The rational unified process: an introduction . Addison-Wesley Professional.

MACHADO, F (2011). Análise e gestão de requisitos de software: onde nascem os sistemas.[sl]: Editora érica;

Ministério da Transparência e Controladoria Geral da União - CGU (2018). Metodologias de gestão de riscos;

Pinto, H. L. M., \& Braga, J. L. (2004). Sistemas legados e as novas tecnologias: técnicas de integração e estudo de caso. Informática Pública, Belo Horizonte, 7 (1), $48-69$.

PMBOK (2018). Guia PMBOK 6 ${ }^{\mathrm{a}}$ Edição - Um Guia do Conhecimento em Gerenciamento de Projetos. PMI.

PRESSMAN, R. S (1995). Engenharia de Software. São Paulo: Makron Books;

Pressman, R. S. (2011). Engenharia de Software - Uma abordagem Profissional. 7 ed., ISBN 978-85-8055-044-3.

Rational IBM. Rational Unified Process (2001) - Best Practices for Software Development Teams. Rational Software White Paper, rev. 11/01.

Reis, C. R., \& de Mattos Fortes, R. (2003). Caracterização de um processo de software para projetos de software livre (Unpublished doctoral dissertation). Universidade de São Paulo.

Schwaber, K., \& Sutherland, J. (2011). The scrum guide. Scrum Alliance, 21.

Sneed, H. M. (2005). Estimating the costs of a reengineering project. In 12th working conference on reverse engineering (wcre'05) (pp. 9-pp). 
SOFTWARE ENGINEERING INSTITUTE - SEI (2010). CMMI® for Development, Version 1.3.

Sommerville, I. (2011). Software engineering 9th edition. ISBN-10, 137035152.

UTFPR (2010). A manutenção de software nas empresas. Disponível em: $<$ http://pg.utfpr.edu.br/dirppg/ppgep/ebook/2010/CONGRESSOS/ADM/25.pdf>, Acesso em: 07 de abril de 2019.

Vogel-Heuser, B., Legat, C., Folmer, J., \& Feldmann, S. (2014). Researching evolution in industrial plant automation: Scenarios and documentation of the pick and place unit (Tech. Rep.).

Weerakkody, V., \& Currie, W. (2003). Integrating business process reengineering with information systems development: issues \& implications. In International conference on business process management (pp. 302-320). 